\title{
Review
}

\section{Bladder Cancer Patient Advocacy: A Global Perspective}

Diane Zipursky Quale ${ }^{\mathrm{a},{ }^{*}}$, Rick Bangs ${ }^{\mathrm{b}}$, Monica Smith $^{\mathrm{c}}$, David Guttman ${ }^{\mathrm{d}}$, Tammy Northam ${ }^{\mathrm{e}}$, Andrew Winterbottom ${ }^{\mathrm{f}}$, Andrea Necchi ${ }^{\mathrm{g}}$, Edoardo Fiorini ${ }^{\mathrm{h}}$ and Stephanie Demkiw ${ }^{\mathrm{i}}$

${ }^{a}$ President and co-Founder, Bladder Cancer Advocacy Network

${ }^{\mathrm{b}}$ BCAN, SWOG, and NCI Patient Advocate

${ }^{\mathrm{c}}$ Executive Director, Bladder Cancer Advocacy Network

${ }^{\mathrm{d}}$ Co-Founder and Chair, Bladder Cancer Canada

${ }^{\mathrm{e}}$ Executive Director, Bladder Cancer Canada

${ }^{\mathrm{f}}$ Founder and Director, Fight Bladder Cancer

${ }^{\mathrm{g}}$ Founder, Associazione PaLiNUro (Pazienti Liberi dalle Neoplasie Uroteliali); Department of Medical Oncology, Fondazione IRCCS Istituto Nazionale dei Tumori, Milano, Italy

${ }^{\mathrm{h}}$ Founder, Associazione PaLiNUro (Pazienti Liberi dalle Neoplasie Uroteliali)

${ }^{\mathrm{i}}$ Founder, Bladder Cancer Awareness Australia

\begin{abstract}
Over the past 20 years, cancer patient advocacy groups have demonstrated that patient engagement in cancer care is essential to improving patient quality of life and outcomes. Bladder cancer patient advocacy only began 10 years ago in the United States, but is now expanding around the globe with non-profit organizations established in Canada, the United Kingdom and Italy, and efforts underway in Australia. These organizations, at different levels of maturity, are raising awareness of bladder cancer and providing essential information and resources to bladder cancer patients and their families. The patient advocacy organizations are also helping to advance research efforts by funding research proposals and facilitating research collaborations. Strong partnerships between these patient advocates and the bladder cancer medical community are essential to ensuring sustainability for these advocacy organizations, increasing funding to support advances in bladder cancer treatment, and improving patient outcomes.
\end{abstract}

Keywords: Bladder cancer, patient advocacy, patient advocate, public awareness, patient education, Bladder Cancer Advocacy Network (BCAN), Bladder Cancer Canada (BCC), Fight Bladder Cancer, Pazienti Liberi dale Neoplasie Uroteliali (PaLiNUro), Bladder Cancer Australia (BCA), improved outcomes, quality of life, partnership

\section{CANCER PATIENT ADVOCACY}

The power of patients to influence, educate, and motivate change to the healthcare landscape has increased significantly in the past two decades. Breast cancer and AIDS activism in the 1980s and 1990s demonstrated the ability of unified voices to catalyze unprecedented changes in public health policy and

\footnotetext{
${ }^{*}$ Correspondence to: Diane Zipursky Quale, E-mail: dzquale@ bcan.org.
}

industry practices. Starting in the late 1990s, rapid adoption of the internet created cohesive health communities despite geographic dispersion - it was easier to find "people like me" than ever before.

Representing these health communities is the role of patient advocacy groups. While cancer patient advocacy organizations may differ in mission and purpose, they share the belief that patient engagement in cancer care is essential to improving patient quality of life and outcomes. In many cancer arenas, partnerships between patient advocacy organizations and medical 
professionals have produced significant benefits to patients and medical professionals alike: improved educational resources and tools empowering patients to be more proactive in their care, improved communications among patients providing essential support through the cancer journey, improved awareness of clinical trials, and increased support for cancer research.

The growth of cancer patient advocacy has not been consistent across cancer types or across the world. Breast, prostate, colon, and lung cancers started the $21 \mathrm{st}$ century with numerous organizations, and their numbers continue to grow. Inconsistency in the maturity of cancer patient advocacy is also seen globally. U.S. advocacy groups are typically the most experienced and successful, with Canadian groups now making similar progress. While European advocacy groups are becoming more prevalent, "the maturity and effectiveness of these groups varies among countries and among cancer types"[1]. In Latin America, "the region has a paucity of viable, active advocacy networks related to cancer control. Furthermore, most groups have very limited experience or even knowledge of patient advocacy, with 'survivorship,' for the most part, emerging as a concept rather than a program reality.... [M] ost groups have little history of advocacy in the local or national policy arena" [2]. Africa and Asia are sadly even less mature.

\section{BLADDER CANCER PATIENT ADVOCACY}

Despite the prevalence of bladder cancer worldwide, bladder cancer patient advocacy got a late start. There was no national bladder cancer patient advocacy organization anywhere in the world until 2005, when the Bladder Cancer Advocacy Network (BCAN) was created in the United States. BCAN (pronounced "beacon") was founded by Diane Zipursky Quale and her late husband, John Quale, a bladder cancer patient who died from the disease in 2008.

Bladder cancer patient advocacy is now expanding around the globe, with non-profit organizations established in Canada, the United Kingdom and Italy, and efforts underway in Australia. Each of these organizations is at a different level of maturity and success. However, the genesis of bladder cancer patient advocacy organizations around the world is universal: a personal experience with the disease compelling action to increase bladder cancer awareness and improve the quality of life for bladder cancer survivors and their families.

In every country, these organizations face the challenge of successfully reaching bladder cancer patients across a broad geographic area. In addition, these organizations share the challenge of continuing to raise the monies needed to provide the educational programming and support that is in great demand in the bladder cancer community. Strong partnering between survivors and the bladder cancer medical community is essential to ensuring sustainability for these advocacy organizations, generating funding to support advances in bladder cancer treatment, and improving patient outcomes.

\section{BLADDER CANCER ADVOCACY NETWORK}

When John Quale was initially diagnosed with bladder cancer in 2000, the Quales, like most newlydiagnosed patients and their families, had never heard of the disease nor did they know anyone else with the disease. As their treatment journey continued, the Quales were surprised to learn of the prevalence of bladder cancer, and dismayed to find out that no treatment advances had been made in more than a generation and, as a result, few treatment options were available. The Quales established BCAN in 2005 as a not-for-profit organization supported by individuals, foundations, and corporations that would create a voice for bladder cancer patients and their families.

BCAN's mission is three-pronged: (1) improve awareness of bladder cancer; (2) provide essential educational tools and resources for patients and their families; and (3) advance research by supporting scientific collaboration and funding bladder cancer research to improve treatments and save lives. To execute this mission, BCAN has a staff of six professionals and more than 300 volunteers across the United States.

From its inception, BCAN has focused on raising awareness of bladder cancer among the general public and the medical community. Through its website, www.bcan.org, as well as its presence on social media, BCAN connects survivors and physicians across the country. Its annual Walkfor Bladder Cancer ${ }^{\circledR}$ in early May focuses on raising awareness in local communities across the U.S. Significantly, BCAN and its team of volunteers successfully moved the United States Senate to pass a resolution in 2015 officially recognizing May as "National Bladder Cancer Awareness Month."

With the oversight of its Scientific Advisory Board of more than 50 bladder cancer specialists, including urologists, medical and radiation oncologists, pathologists, social scientists, and basic scientists, BCAN provides a variety of educational tools for bladder cancer survivors. The Scientific Advisory Board ensures 
the accuracy of information provided by BCAN and alignment with critical scientific goals.

In 2008, BCAN first published Bladder Cancer Basics for the Newly Diagnosed, a comprehensive guide on bladder cancer diagnosis and treatment options that helps patients make informed choices about their care. Revised in 2012, this online and print resource is available in both English and in Spanish, and has been widely distributed to urology practices and medical centers, with more than 250,000 copies in circulation.

BCAN also engages bladder cancer specialists to lead its quarterly Patient Insight Webinar series, which has covered topics such as personalized medicine, clinical trials, and bladder cancer tests, diagnosis and recurrence. BCAN recently launched a video series, "The New Normal: Living with a Urinary Diversion," featuring bladder cancer survivors discussing life after a radical cystectomy. In addition, BCAN has received funding from the Patient-Centered Outcomes Research Institute (PCORI) to actively engage bladder cancer patients and caregivers in research prioritization by developing a "patient survey network."

BCAN has encouraged the development of support groups in communities across the U.S. BCAN's online support community, launched in 2007, now has over 12,000 members, connecting bladder cancer survivors with peers around the world. In addition, BCAN's Survivor 2 Survivor program connects the newly diagnosed patient with a more "seasoned" patient who has undergone similar treatment and can provide firsthand accounts of life with bladder cancer.

BCAN's educational efforts have also focused on informing patients about clinical trials and the important role these studies play in developing effective new treatments for bladder cancer. Working with a group of urologists and medical oncologists, BCAN has developed its "Clinical Trials Dashboard," a resource for patients and physicians to find a current listing of open bladder cancer clinical trials.

One of BCAN's earliest research accomplishments was the establishment of its annual Bladder Cancer Think Tank meeting, which focuses on identifying obstacles and creating solutions in bladder cancer research. Started in 2006, the meeting is a strong catalyst for scientific collaboration, and has fostered discussions that have defined priorities for bladder cancer research.

BCAN has also advanced bladder cancer research by directly funding bladder cancer researchers. Through its John Quale Travel Fellowship Awards, its Young Investigator Awards, and its Bladder Cancer Research
Innovation Awards, BCAN has provided $\$ 1.5$ million in direct research funding. These programs are vital to attracting clinicians and researchers to focus on bladder cancer. The ability of individual institutions and individual researchers to advance the science of any disease is limited today, and the complexity of cancer makes collaboration even more critical. Patient advocacy organizations like BCAN provide the impetus as well as the mechanism - to promote much-needed cooperation and create synergy.

\section{BLADDER CANCER CANADA}

Bladder Cancer Canada (BCC) was formed in 2009 by bladder cancer survivors David Guttman and Jack Moon, who at the time of initial diagnosis, had never heard of bladder cancer and didn't know anyone else with the disease. While Mr. Guttman and Mr. Moon found support through BCAN, they saw a need for a Canadian-specific organization due to the differences in the countries' respective health care systems. This Canadian national charity was organized to: (1) help bladder cancer patients and their support teams address the day-to-day issues of this disease; (2) increase awareness of bladder cancer among the general public and medical community; and (3) fund research which pursues the diagnosis, treatment, and elimination of bladder cancer. BCC does not have an office, but does have a professional Executive Director. The organization relies heavily on volunteers all across Canada to help advance its mission, and is funded primarily through donations, bequests and some corporate support.

Like BCAN, BCC was launched with the strong support of the medical community. There are now 28 bladder cancer specialists serving on BCC's Medical Advisory Board and/or Medical Research Board. Canadian physicians have come to rely on BCC as a credible and valuable place to send their patients for support and information. Its website, www.bladdercancercanada.org, provides much-needed educational and support resources for the Canadian bladder cancer community. The website includes an active online discussion forum.

$\mathrm{BCC}$ has been very successful in its early years sponsoring patient educational meetings in cities across Canada. The organization has also worked to develop local patient support groups, and hosts its own online support community. Most recently, BCC published two patient guidebooks: one for patients facing radical cystectomy and one for patients with non-muscle 
invasive bladder cancer. These patient-centered resources, created by patients and their caregivers, are available online and in hard copy.

In the area of medical research, Bladder Cancer Canada established and provides funding for the Canadian Bladder Cancer Information System (CBCIS) to improve the management of bladder cancer patients across Canada by serving as the Canadian multidisciplinary and multicenter platform and resource. The CBCIS will capture contemporary management, practice patterns, and outcomes of the entire spectrum of bladder cancer in Canada. Initial focus will be on nonmuscle invasive bladder cancer with a later focus on muscle invasive bladder cancer. BCC is also providing funding for the Quality of Care Initiative, a new effort by the BCC Medical Research Board to develop national bladder cancer quality of care standards. The initiative launched in 2014 with a meeting of 25 bladder cancer experts who discussed bladder cancer quality of care standards. Future meetings are anticipated.

BCC has been extremely successful in raising awareness across Canada through its annual Awareness Walk in September. The walk, held in more than 20 cities annually, raises in excess of $85 \%$ of BCC's annual revenue. In addition, since 2013, BCC has implemented a public awareness campaign called "See Red, See Your Doctor." By leveraging relationships with many of the larger Canadian advertising suppliers, BCC was able to secure approximately $\$ 2$ million worth of advertising for only $\$ 100,000$ which was seen as posters in transit shelters, subway and commuter railway stations, underground walkways, inside and outside buses, on full size billboards, and in movie theaters, restaurants, and exercise facility washrooms. BCC also had ads in various magazines. In addition, $\mathrm{BCC}$ had videos nationwide on hundreds of screens in doctors' offices, highway stops, hospital waiting rooms, and movie theater lobbies, with tens of millions of potential viewers. The "See Red" public service announcement has also been promoted through donated spots on national television.

\section{FIGHT BLADDER CANCER}

Andrew Winterbottom was diagnosed with Stage 4 bladder cancer in 2009, after initially being misdiagnosed with an enlarged prostate. He was surprised to find no charity or advocacy group dedicated to bladder cancer in the United Kingdom, despite the prevalence of the disease. He found great support from another bladder cancer survivor, and understood the need to provide that kind of support to others. Fight Bladder Cancer is a registered charity in the UK with three primary objectives: (1) support everyone affected by bladder cancer, including family and friends of the patient; (2) raise awareness of the disease, so that people can be diagnosed at the earliest possible time; and (3) increase research for bladder cancer by working with research funding organizations to help ensure that a larger amount and proportion of research dollars are targeted to bladder cancer.

Fight Bladder Cancer initially started as a local support group and "unregistered charity," but it soon became clear that the lack of available support for bladder cancer survivors and their families was a national problem, and needed to be tackled on a much wider scale. The first step for these committed volunteers was to set up Bladder Cancer Support UK as a Facebook forum in 2010. This encouraged people to engage with other people affected by bladder cancer honestly and openly, and the group quickly grew to its current $1700+$ members.

Once the support group was successful, Mr. Winterbottom set up a Twitter feed to spread the word further and start campaigning for better bladder cancer awareness. Fight Bladder Cancer now has close to 2,000 Twitter followers across the globe, including many leading urologists. In 2013, the organization launched its own dedicated website for Fight Bladder Cancer which has proven very popular with patients across Europe and around the globe, www.fightbladdercancer.co.uk. This was linked with the design and publication of awareness leaflets and patient support material to be handed out by doctors and nurses in hospital clinics. Fight Bladder Cancer was registered as a UK charity in 2014 and began to publicly solicit donations.

Fight Bladder Cancer helps to set up local support groups around the UK, and provides a one-to-one Bladder Buddy Service where newly diagnosed patients or their caregivers are put in touch with someone local who has had the same diagnosis and treatment so that they can have someone to whom they can talk to get much needed support.

Fight Bladder Cancer also now partners with the bladder cancer medical experts, many of whom provide assistance with their educational materials and awareness efforts. This panel of support is growing larger and more robust across all the specialties. The charity is now represented on the national Bladder Cancer Clinical Studies Group which helps to manage the portfolio of clinical studies in the UK and is part of the panel developing the National Institute for Health and 
Care Excellence (NICE) Bladder Cancer Quality Standards. Fight Bladder Cancer is now supporting specific research on bladder cancer issues with a current special emphasis on Quality of Life issues.

The organization recently joined two other UK nonprofit organizations in the "Shout Out About Bladder Cancer" campaign, a united effort "to help bring a much needed spotlight to this very common, but hardly talked about cancer."

\section{PALINURO}

PaLiNUro (Pazienti Liberi dalle Neoplasie Uroteliali, in English "Patients Free from Urothelial Tumors") was started in Milan, Italy in 2014 at the Fondazione IRCCS Istituto Nazionale dei Tumori by a group of patients and doctors who recognized the need for bladder cancer survivors to provide support for one another, and the need to increase the publicly-available information to the urologic and oncologic communities about bladder cancer and all its clinical needs. PaLiNUro aims to give newly-diagnosed patients precise, yet practical, answers, filtered through the voice of other experienced patients and addressing the challenges that arise from a bladder cancer diagnosis. Another major aim of PaLiNUro is to promote a new path for clinical research to include patient advocates in the design of clinical trials for bladder cancer. Advocate participation will be essential to ensuring well-designed trials that will enroll quickly, given the increasing number of clinical studies with novel compounds, often in a multidisciplinary setting involving clinical oncologists and urologists.

PaLiNUro is currently an all-volunteer organization. The organization's website, www.associazionepalinuro.com, already provides important resources for bladder cancer patients. The group is working diligently to find more volunteers, as well as financial supporters, who can contribute to PaliNUro's nationwide activities to support research in the diagnosis and treatment of bladder cancer. Specifically, PaliNUro is organizing patient meetings on hot topics like sexuality after radical cystectomy, distributing leaflets and brochures on the aims and activities of the group, and organizing recreational activities to bring people together and provide information on the organization.

PaliNUro is also working to create a European patient advocacy alliance for bladder cancer. The organization recently joined the European Cancer Patient Coalition to help facilitate this broader alliance.

\section{BLADDER CANCER AWARENESS AUSTRALIA}

Stephanie Demkiw of Melbourne, Australia was a surgical trainee when her father was diagnosed with metastatic bladder cancer. No one in her family knew anything about the disease, and the family was surprised at the lack of information and resources available, as well as the absence of any organization or charity focused on patients with the disease. Dr. Demkiw's father died in 2013, and her family has launched BCA, Bladder Cancer Awareness Australia, to raise awareness about the disease, provide educational materials to survivors and their families, and support research.

Currently operating as a social media community, including Facebook and Instagram, BCA has initiated campaigns to raise awareness about hematuria, with the popular hashtag "\#loveyourbladder" and is partnering with Cancer Council Australia to help connect bladder cancer patients around the country. BCA was officially registered for charity status in April 2014, and will be launching its website in the coming months. The major fundraising and awareness events in 2015 are Australia's Biggest Morning Tea and The Pelvic Ball, a black tie event in association with Ovarian Cancer Awareness. Dr. Demkiw intends to recruit additional physicians and researchers to the organization through presentations at upcoming national medical conferences.

\section{THE FUTURE OF BLADDER CANCER PATIENT ADVOCACY}

Bladder cancer patient advocacy, while not yet thriving, is certainly growing, both in numbers and maturity. In the ten years since 2005, longstanding voids for US, Canadian, UK, Italian, and Australian bladder cancer patient advocacy have been filled. However, the absence of bladder cancer advocacy in other parts of Europe, as well as in Africa, Asia, and the Middle East, leaves large disparities in public awareness of the disease, as well as essential information, support, and treatment options for bladder cancer patients globally. We hope that patient advocates and medical professionals in other countries will be encouraged to leverage the experiences of the groups that exist today and move to fill these gaps.

The exciting advances finally being made in bladder cancer - in part due to the success of bladder cancer patient advocacy - present significant opportunities 
and some challenges. Bringing new treatment options from "bench to bedside" will require research funding and willing participants in clinical trials. Accrual to clinical trials in bladder cancer has always been extremely challenging, sometimes deterring research in this space. The need for education by advocacy groups about clinical trials in general and new treatment options outside of traditional chemotherapy and radiation therapy continues to grow. The strong partnership between bladder cancer advocacy groups and the medical community must be leveraged to achieve acceptable rates of clinical trial participation.

At the same time, researchers will need patient advocates to partner with them as they design, develop, and deliver these clinical trials. But developing skilled patient research advocates will require training and on-the-job experience. Formal training programs like those available in the breast and other cancer communities can ensure a pipeline of qualified candidates is available to meet increased demand.

Sustained momentum for BCAN, BCC, Fight Bladder Cancer, PaLiNUro, and BCA requires strong partnerships between the advocates and the bladder cancer medical community. Each of these organizations relies on reaching bladder cancer survivors from across its respective country to provide volunteer service as well as philanthropic funding to fulfill its mission. One critical pathway to reaching bladder cancer survivors is through the urologists, medical oncologists, radiation oncologists, nurses, and other professionals who are treating them. By working with these advocacy organizations, the bladder cancer medical community can greatly accelerate increased awareness of bladder cancer, educational and support resources, and support for bladder cancer research - resulting in improved prognosis, treatment options, and quality of life for bladder cancer patients across the world.

\section{REFERENCES}

[1] Redmond K, Collaboration with patient advocates: Unleashing a potent force. BJU Int 2003 May;91(7):590. Available: http://onlinelibrary.wiley.com/doi/10.1046/j.1464-410X.2003. 04210.x/pdf

[2] Durstine A, Leitman E, Building a Latin American cancer patient advocacy movement: Latin American cancer NGO regional overview. Salud publica de Mexico; 51, suplemento 2 de 2009: 321. 